\title{
Circuit
}

Musiques contemporaines

\section{Dossier de création : Re Orso (2012) de Marco Stroppa}

\section{Serge Provost et Cristina Scuderi}

Volume 23, numéro 1, 2013

URI : https://id.erudit.org/iderudit/1017213ar

DOI : https://doi.org/10.7202/1017213ar

Aller au sommaire du numéro

Éditeur(s)

Les Presses de l’Université de Montréal

ISSN

1183-1693 (imprimé)

1488-9692 (numérique)

Découvrir la revue

Citer cet article

Provost, S. \& Scuderi, C. (2013). Dossier de création : Re Orso (2012) de Marco

Stroppa. Circuit, 23(1), 69-75. https://doi.org/10.7202/1017213ar d'utilisation que vous pouvez consulter en ligne.

https://apropos.erudit.org/fr/usagers/politique-dutilisation/ 


\section{ACTUALITÉS}

\section{Dossier de création : \\ Re Orso (2012) de Marco Stroppa}

Serge Provost et Cristina Scuderi

\section{I) L'opéra réincarné : compte rendu de Re Orso}

Légende musicale de Marco Stroppa ${ }^{1}$, pour quatre chanteurs, quatre acteurs, ensemble, voix et sons imaginaires, spatialisation et totem acoustique.

Livret de Catherine Ailloud-Nicolas et Giordano Ferrari d'après Arrigo Boito².

Création le 19 mai 2012, Opéra Comique, Paris.

Direction musicale, Susanna Mälkki, Ensemble intercontemporain.

Informatique musicale, Ircam, Carlo Laurenzi.

Mise en scène, Richard Brunel.

Avec Rodrigo Ferreira (Re Orso/Roi Ours), Monica Bacelli (le Ver), Marisol Montalvo (Oliba), Alexander Kravets (le Trouvère), Geoffrey Carey (Papiol), Cyril Anrep, Daniel Carraz, Piera Formenti (des courtisans).

La création de Re Orso pourrait inaugurer une période de mutation importante de ce qu'il est convenu d'appeler l'opéra lyrique, non seulement par l'intégration de nouvelles technologies, mais également par une redéfinition de la relation entre théâtre et musique.

C'est le musicologue Giordano Ferrari qui soumettra les poèmes de Boito, dont le Re Orso - cette fable à propos du pouvoir et de ses excès - séduira le compositeur par ses qualités littéraires, sa musicalité et son engagement politique. Marco Stroppa se définit d'ailleurs lui-même comme un altermondialiste engagé.

L'œuvre se présente en deux parties:

I. Orso vivant:

À la cour de Crète, il y a plus de mille ans, règne un tyran sanguinaire qui entraîne sa cour dans la démesure. Ayant commis un meurtre sous le regard de ses courtisans, il les contraint à célébrer ses méfaits. Toutefois, la même nuit, une voix se manifeste et hante Re Orso: serait-ce la voix de celle qu'il a tuée? Non, il s'agit de la voix de sa conscience qui le ronge comme un ver, ce dernier étant représenté par un personnage féminin. Re Orso prend alors pour épouse Oliba, qui se refuse à lui. La voix continue de le hanter. Fou de rage, il tue son épouse, le Trouvère et le Ver.

\section{Ours mort:}

Le roi mourant tente de racheter son salut, mais le Ver revient avec toutes les victimes de la première partie et devient la voix du peuple qui vaincra le tyran. 


\section{Lieux orgiaques (première partie)}

Au lever du rideau apparaît un espace luxueux, stylisé, le jardin du Roi Ours. Nous pourrions évoquer un espace fellinien revampé...

Les sept scènes qui composent cette première partie sont autant de jalons qui, du meurtre initial d'une femme, conduisent le Roi Ours à la déchéance morale la plus profonde. Chaque scène est un tableau qui compose un espace caractéristique animé par des figures, des Leitgestalten (marqueurs perceptifs), qui associent des éléments musicaux, sonores, à un caractère, une situation. De plus, chaque voix est associée à un instrument et une figure de danse - par exemple le tango et le registre aigu du basson pour souligner le caractère efféminé mais aussi bestial du roi. Ainsi la succession des scènes se décline selon le schéma suivant:

- Scène 1: Histoires anciennes ébouriffées (passacaille rocky avec verve) $)^{3}$. On y trouve une table pour le banquet, une plateforme de pierre, lieu du meurtre de la jeune femme. Après le crime, la terre s'entrouvre, les flammes jaillissent et la plateforme-tombeau s'enfonce dans les profondeurs.

- Scène 2: Spectrum (pompeux, décomplexé). Une voix mystérieuse hante Re Orso. Est-ce la voix de la femme qu'il a tuée ou celle de sa conscience qui le ronge, tel un ver?

- Scène 3: Sérénade de pierre (loufoque). Re Orso veut s'unir à Oliba qui se refuse à lui. Il tente de la violer avec ses courtisans.

- Scène 4: Duel dialectique (embrouillé) et larve de tango (émietté). La voix s'incarne: il s'agit du Ver. Dialogue dialectique.

- Scène 5: Noces et chansons (outrecuidant) et intermède historique (festif, chaotique). On célèbre les noces du roi et d'Oliba. Un trouvère est présent avec son double, un piano robotisé.
En proie à la colère, à la folie, Re Orso tue le Trouvère, Oliba et le Ver. Les musiciens quittent précipitamment la fosse d'orchestre et s'enfuient en jouant encore quelques notes.

L'enchaînement presque frénétique des scènes, les caractères très marqués, les mouvements scéniques de plus en plus nerveux, la «machine " que constitue le lit de mort qui s'enfonce dans les ténèbres, les personnages magiques (le Trouvère vs le piano robotisé, le Ver réincarné) semblent tirer leur source de l'ancêtre lointain : l'opéra baroque, avec ses décors, ses machines descendant des cieux, ses personnages mythologiques et, surtout, la représentation d'un spectacle magique et fabuleux. Giordano Ferrari précisera que, dans ce contexte, les personnages sont avant tout des symboles, des porteurs de sens qui s'affrontent dans un espace intemporel ${ }^{4}$.

\section{Lieux funestes (seconde partie)}

La déchéance du roi et l'avènement du peuple libéré sont relatés en trois scènes:

- Scène 1: Confession (rugueux, obsolète). Les morts réapparaissent et le Ver retrouve le roi.

- Scène 2: Litanie. grand tutti bordélique (grand pandémonium, comme une gigue boiteuse), qui accompagne la mort, sans rédemption, du roi.

- Scène 3: Pierre tombale, cercueil, linceul (serein, sérieux, simple sûr). Le Ver, devenu voix du peuple, voix du poète, célèbre sa victoire sur le tyran.

Toute cette seconde partie est consacrée au Ver réincarné dont la voix subtilement mutée en sons invisibles, magique comme une aura lumineuse, est accompagnée de l'accordéon qui parle grâce à un procédé de synthèse sonore. Enfin, s'ajoute un totem acoustique, formé d'une colonne de haut-parleurs et qui tourne lentement, diffusant le son comme une brise délicate et lumineuse. Ici, la musique traitée par l'électronique 
devient un paradigme en soi avec une authentique présence poétique.

\section{Post-scriptum}

Une grande force de cette œuvre réside dans la remarquable synthèse de tous les éléments se transformant en énergie qui se déploie à travers les éléments. Le son, la lumière, l'action sont animés de mouvements dynamiques constants qui se reconfigurent au gré de la température de l'action globale et de ses ramifications. Ces mouvements sont généralement très articulés et d'une grande vivacité, en particulier dans la première partie.

Les mouvements de la pensée, de l'expression des images, des éléments de forme et de langage, créent des ensembles sémantiques d'où émerge un métalangage multidimensionnel qui se transmute du verbe vers l'espace vibratoire: sons, lumière, intensités, vélocités, toutes reconfigurations ancrées par les marqueurs de forme, Leitgestalt.

Il est clair qu'une intégration réfléchie de la technologie, en particulier du traitement sonore, constitue un vecteur puissant de la cohésion de l'œuvre en extrapolant de la matière tangible une mutation poétique, des espaces imaginaires de pure poésie. Une aura mystérieuse, hors temps, qui enveloppe de sa lumière le lieu tout entier du drame.

Serge Provost

\section{II) Entretien avec Marco Stroppa}

En 2012, la production musicale de Marco Stroppa s'est enrichie d'une œuvre importante, fruit d'un mois de travail intense et d'un dévouement total. Le domaine de l'opéra contemporain accueille ainsi un nouvel opus riche de significations et d'alchimies sonores, miroir de la personnalité tout aussi abondante du compositeur qui, rappelons-le, est également un enseignant réputé, professeur et chercheur partagé depuis plusieurs années entre l'Institut de recherche et coordination acoustique/musique (Ircam) de Paris et la Hochschule für Musik und Darstellende Kunst de Stuttgart 5 .

Après une vive évolution artistique amorcée au début des années 1980 avec les compositions Traiettoria (1982-1984, rev. 1988) et Metabolai (1982), et perpétuée par d'autres œuvres instrumentales, pour orchestre, ensemble ou soliste, des pièces de musique de chambre, notamment de l'«électronique de chambre », deux pièces radiophoniques, plus récemment des pièces chorales, et ce, toujours selon une production éclectique en équilibre entre la composition traditionnelle et la musique pour ordinateur, le parcours de Marco Stroppa a abouti à un premier travail pour le théâtre musical, Re Orso, créé en mai 2012 à l'Opéra Comique de Paris et chaleureusement accueilli par le public de la salle Favart. «Légende musicale pour 4 chanteurs, 4 acteurs, 11 instruments, voix et sons invisibles, spatialisation et totem acoustique » sur un livret de Catherine Ailloud-Nicolas et Giordano Ferrari d'après Arrigo Boito, l'œuvre a été réalisée conjointement avec le metteur en scène Richard Brunel et interprétée par l'Ensemble intercontemporain sous la direction de Susanna Mälkki.

L’opéra comporte deux actes. Le premier, «Orso vivo », raconte les méfaits d'un roi violent, Re Orso, entouré d'une cour servile et effrayée. Être effroyable et meurtrier, il force son épouse au mariage et tente de la violer. Le second acte, «Orso morto», dépeint la chute du roi : ce dernier meurt tourmenté par les fantômes de ses victimes en demandant en vain le pardon. À la fin, le Ver, représenté par une femme du peuple, fête son triomphe sur le cadavre du roi.

Chaque personnage est associé à une danse et un instrument: le roi au basson dans son registre aigu; Oliba, son épouse, à la clarinette; le personnage-clé, le Ver, à l'alto, tandis que le Trouvère, associé à la trompette, possède également un double mécanique, un piano robotisé. Les sons de synthèse interagissent avec 
FIGURE 1 Acte II, scène 2, "Litanie, grand tutti bordélique". Mort de Re Orso. Opéra Comique de Paris, 19 mai 2012 (C)Elisabeth Carecchio).

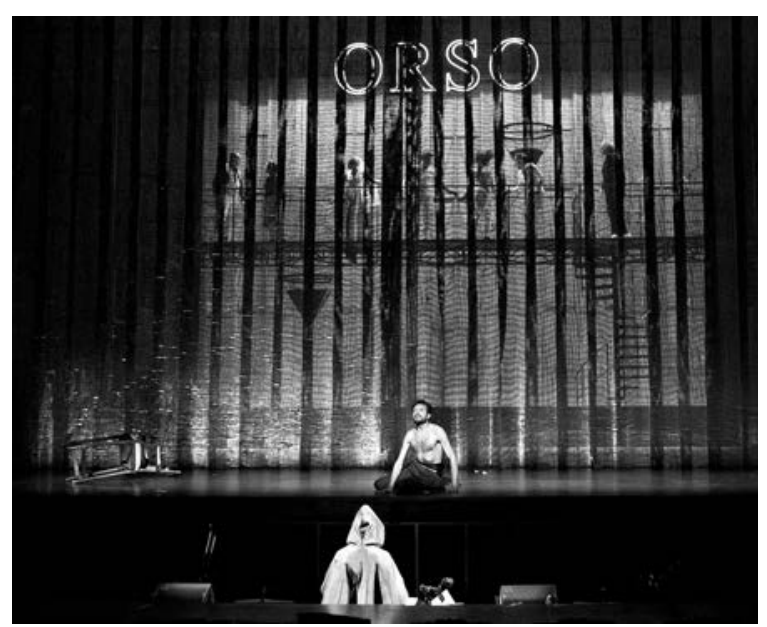

FIGURE 2 Acte I, scène 5, "Noces et chansons". Scène du toast. Opéra Comique de Paris, 19 mai 2012 (C)Elisabeth Carecchio).

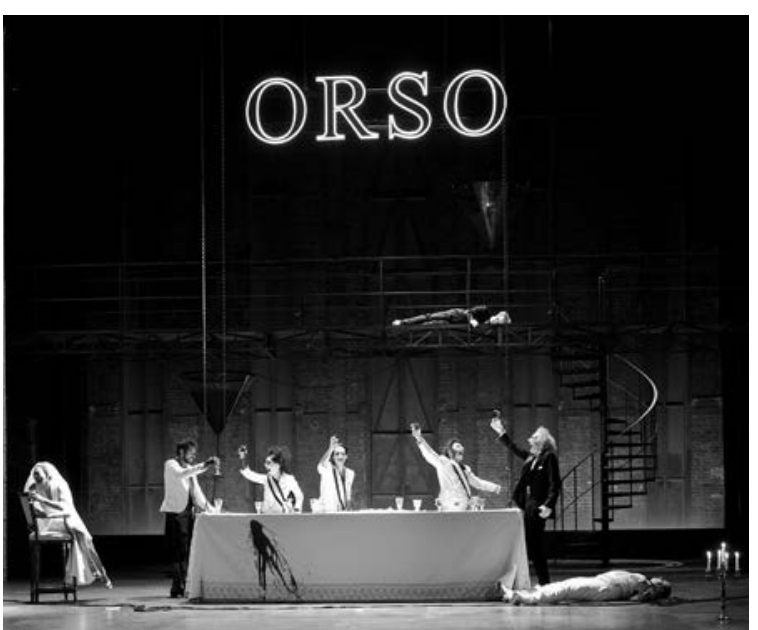

les sons, amplifiés, des voix réelles, choisies dans les tessitures de soprano, mezzo-soprano, contre-ténor et ténor, et des instruments (bois, cuivres, cordes, contrebasse jazz et accordéon). Ces derniers prédominent dans le premier acte alors que le second se consacre principalement à la voix et à l'électronique.

L'opéra fait entendre une contrebasse exploitant les techniques du jazz, un accordéon qui "parle», des rythmes de tango, sarabande, mazurka, ragtime, une citation de Rigoletto et de la Traviata (une parodie du célèbre toast «Libiamo ne’ lieti calici»), une allusion au Don Juan de Mozart, ainsi que des références aux œuvres de Jean-Claude Risset (Songes, 1979), John Chowning (Turénas, 1972) et Mauro Graziani (L'espace entre les pierres, 2008) $)^{6}$. Il s'agit donc d'un kaléidoscope de renvois comportant plusieurs niveaux de lecture et une dimension politique que la fable originale de Boito ne proposait pas. Qui connaît Marco Stroppa sait que sa maturité artistique résulte d'une profondeur de perspective rare, un univers précieux d'intérêts cultivés avec la vive curiosité d'un savant, intérêts qui vont de la psychologie cognitive à l'intelligence artificielle, de l'étude de l'espace sonore à l'ethnomusicologie, de l'amour pour la parole à la poésie et au mythe. Tous ces parcours se réunissent dans Re Orso, qui est, plus qu'un point de repère, un point de départ, grâce aux nombreuses réflexions que l'œuvre suscite.

Nous avons contacté le compositeur pour un bref entretien que nous reproduisons ci-après. Nous sommes à Stuttgart, le 12 novembre 2012.

Cristina Scuderi : Maestro, votre Re Orso, très attendu, a eu une longue et intense gestation, aboutissant à une création acclamée. Vous arrive-t-il de composer en pensant au public et, en particulier, vous êtes-vous déjà interrogé quant à la réception de votre premier opéra?

Marco Stroppa : Richard Brunel et moi nous sommes constamment posé la question des niveaux de compré- 
hension de notre projet de la part d'un public amateur, qui n’est pas nécessairement familier avec la musique contemporaine. Nous avons décidé de proposer plusieurs «lignes» parallèles et simultanées en relation dialectique constante. Par exemple, si la mise en scène crée un «fil rouge» indiquant la direction principale de l'œuvre, la musique tisse des va-et-vient multiples autour de ce fil, tels des courts-circuits temporels (prémonitions ou mémoires variées, fuites hors du temps scénique, etc.). À chaque ligne, il y a une réception possible.

c. s.: Du point de vue musical, y a-t-il dans Re Orso des éléments de vos précédentes créations?

M. S. : J'ai écrit deux opéras radiophoniques sur support: Proemio, en 1990, et In cielo in terra in mare, en 1992. Cependant, leur «logique» interne n'était pas celle d'un «Hörspiel » mais celle d'un véritable opéra, joué sur une scène imaginaire avec plusieurs acoustiques constamment variées, ce qui est impossible à réaliser dans un théâtre. Cette expérience m'a guidé dans l'écriture de Re Orso bien qu'elle n'ait pas été directement utilisée.

c. s. : Que retrouve-t-on de votre esprit et de vos sentiments les plus profonds dans votre nouvel opéra?

M. s. : Beaucoup d'aspects parmi lesquels la critique de toute situation de pouvoir et d'oppression, à n'importe quel niveau - de la relation de couple à la famille, d'un groupe à la société -, l'espoir que la «voix du peuple », représentée par le Ver dans l'opéra, finira par l'emporter, et l'utilisation de Leitgestalten, à savoir une sorte de «balisage» cognitif des principales situations dramaturgiques, une technique dérivée de ma pratique compositionnelle instrumentale et plus évoluée que celle du leitmotiv wagnérien?

La Leitgestalt peut être considérée comme une extension, dans le domaine de la dramaturgie musicale, du concept d'organismes d'information musicale
(OIM) que j’ai élaboré au milieu des années $1980^{8}$. Il s'agit de morphologies particulières et extrêmement variées, basées sur une hiérarchie de dimensions ou de relations entre dimensions, et dont la fonction est de souligner certains éléments de la dramaturgie. Par exemple, la voix du Ver est toujours accompagnée par un traitement électronique particulier, appelé «delay spectral », qui renforce le sens de ce qui est chanté ou le rôle dramaturgique du personnage, selon différentes modalités. Ce concept est encore assez nouveau pour moi. Je pense écrire un texte, un jour, pour le formaliser de façon plus précise. Mais pour l'instant, je l'exploite un peu intuitivement pour en faire l'expérience!

C. s. : Pourriez-vous décrire la construction de l'œuvre en quelques mots?

M. $\mathbf{s}$. : Le «germe» de «l'ours» a été planté dans mon esprit par le musicologue Giordano Ferrari qui savait que depuis mes opéras radiophoniques je cherchais l'occasion d'écrire à nouveau pour l'opéra sans en trouver le contexte adéquat. Un jour, Giordano m’a donné à lire quelques textes d'Arrigo Boito parmi lesquels Re Orso, une histoire en vers polymétriques écrite par l'auteur à 21 ans. Construit comme une œuvre musicale, avec des thèmes ou des mots récurrents, ce poème fait preuve d'une imagination linguistique sensationnelle et m'a tout de suite interpellé.

Nous avons ainsi demandé à un dramaturge italien expérimenté d'écrire un livret, mais le résultat fut tellement problématique que nous avons été contraints, Richard Brunel et moi, ainsi que les deux auteurs du livret final, Catherine Ailloud-Nicolas et Giordano Ferrari, de repartir à zéro.

Pendant deux mois, nous nous sommes vus régulièrement afin de concevoir ensemble un spectacle complet, en planifiant les coups de théâtre, l'équilibre des scènes, le sens à transmettre - par exemple, comment représenter le Ver qui, dans le texte de Boito, est un véritable lombric, mais ne pouvait évidemment pas 
FIGURE 3 Acte II, scène 3, "Pierre tombale, cercueil, linceul". Le triomphe du Ver; au centre, le totem acoustique. Opéra Comique de Paris, 19 mai 2012 (C) Elisabeth Carecchio).

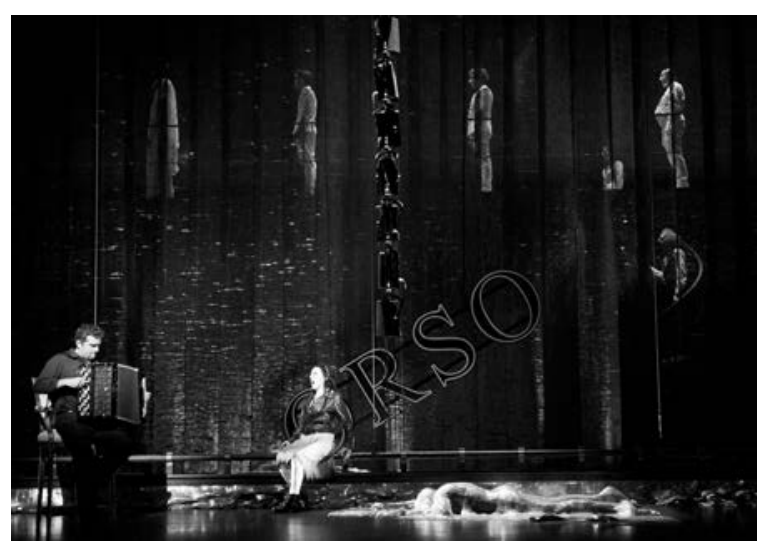

être mis en scène en tant que tel! Pendant cette phase passionnante, chacun a apporté ses idées en partant des bases que j’avais établies: par exemple, le fait que l'ensemble doit quitter la scène à la fin du premier acte - le deuxième acte comporte uniquement la voix et l'électronique - ou l'apparition, à la fin, d'un «totem acoustique », soit une colonne de huit haut-parleurs suspendue au milieu de la scène.

Pendant cette phase, j'ai construit au fur et à mesure une sorte de story-board détaillé où tous ces éléments ont été écrits et minutés avec une précision d'une dizaine de secondes. Dans ma tête, l’opéra était composé. Il ne restait plus qu’à l'écrire!

c. s.: Dans une précédente entrevue, vous dites que le compositeur est nécessairement un «chercheur». La musique, la technologie et la science se rejoignent dans Re Orso, conduisant à des expédients inusités dans l'opéra contemporain. À quelles nouveautés musicales votre œuvre a-t-elle conduit les recherches menées auprès de l'Ircam?

M. s.: On remarquera que le terme «électronique» ne figure pas dans le sous-titre, remplacé par les rôles qu'elle joue dans la dramaturgie de l'œuvre, qui sont du même niveau que ceux des autres personnages et doivent, in fine, parvenir à émouvoir le public et raconter une histoire.

Pour y arriver, cependant, nous avons dû faire parfois beaucoup de recherche, en particulier en ce qui concerne la synthèse d'une voix chantée chimérique. Développée à l'Ircam au début des années 1980, la synthèse de la voix chantée n'a pas survécu aux changements des environnements informatiques et n'était donc plus utilisable.

Nous avons retrouvé les règles développées à l'époque, analysé des parties de l'ancien code informatique, discuté avec Xavier Rodet, qui avait dirigé ce projet, et intégré le tout - j'espère en mieux! - dans OpenMusic, un environnement d'aide à la composition développé par Jean Bresson, le conseiller scientifique de Re Orso.

Avec Carlo Laurenzi, le réalisateur en informatique musicale qui a travaillé avec moi, et grâce à l'aide du scientifique Arshia Cont, nous avons également développé un «delay spectral» particulier, à savoir un algorithme permettant de décomposer le spectre d'un son en des centaines de petites «tranches », appelées bins, puis de retarder chaque tranche individuellement selon un contrôle harmonique et rythmique très précis. C'est le traitement principal de la voix du Ver. Nous avons dû aussi trouver le moyen de faire «parler» un accordéon en n'utilisant que le jeu de l'instrument qui doit « réciter » quelques vers du livret dans la dernière scène.

Enfin, les personnages du Trouvère et de Papiol, le bouffon décalé de la cour, ont un double mécanique: un piano robotisé pour le premier et une grande marionnette pour le second. Véritable personnage pourvu de sentiments, ce piano est contrôlé par un logiciel entièrement nouveau, l'Antescofo, développé par l'équipe dirigée par Arshia Cont et qui non seulement suit le tempo du chef, mais permet également 

bouffon Papiol. Opéra Comique de Paris, 19 mai 2012 (C) Elisabeth Carecchio).

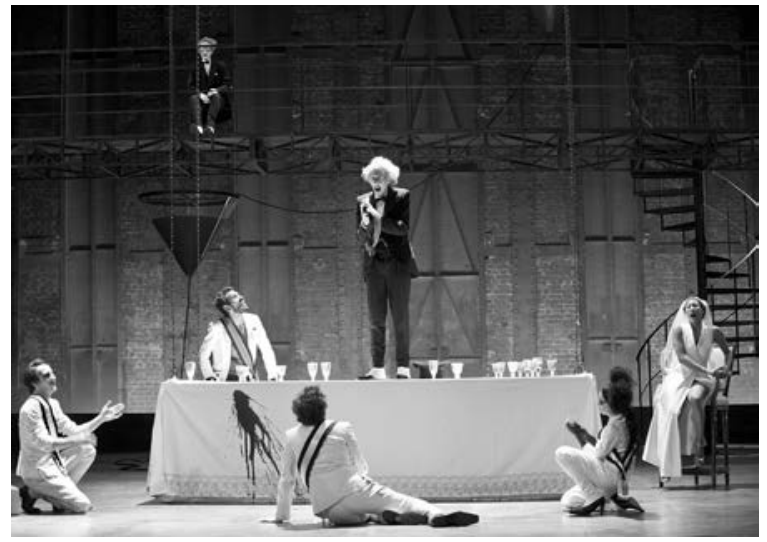

d'écrire une «partition» des gestes de l'instrument qu'aucun interprète ne pourra jamais jouer ainsi, c'està-dire avec une précision et une puissance équivalentes à celles d'un langage informatique. Et comme son double «humain », le piano meurt sur scène à chaque représentation.

c. s.: Dans un avenir prochain, pensez-vous réaliser une autre œuvre opératique? Y a-t-il un texte sur lequel vous aimeriez travailler?

M. S.: Je ne crois guère à un modèle productiviste, que ce soit dans l'agriculture ou dans la composition! Re Orso a certes reçu un accueil très positif, mais ce n'est pas une raison pour écrire d'autres opéras. Les circonstances qui m’ont amené à le composer ont été exceptionnelles: une synergie particulière, peut-être unique, s'est créée entre nous. Si je parviens à la retrouver, alors l'hypothèse d'un autre opéra pourra être envisagée. Cependant, je me concentre pour le moment sur la reprise de Re Orso à Bruxelles, en septembre 2015: il y encore tellement de détails que je souhaiterais améliorer dans la réalisation informatique!

Cristina Scuderi
1. Marco Stroppa est un compositeur italien né le 8 décembre 1959 à Vérone. En 1982, à la demande de Pierre Boulez il s'installe à Paris, où il travaille comme compositeur et chercheur à l'Ircam. Depuis 1999, il est professeur de composition à la Musikhochschule de Stuttgart, succédant à Helmut Lachenmann.

2. Arrigo Boito (1842-1918), né à Milan, est un compositeur, romancier italien, connu surtout pour ses livrets d'opéra et pour son drame Iyrique, Mefistofele.

3. Ces informations proviennent du programme distribué par l'Opéra Comique lors des représentations, disponible en ligne: <www.operacomique.com/wp-content/uploads/2012/05/Re-Orso-a-lire-avant-lespectacle.pdf> (consulté le 19 février 2013).

4. Ibid.

5. Pour de plus amples informations sur Marco Stroppa et son œuvre, I'on pourra consulter le site Internet du compositeur: $<$ www.marcostroppa.eu $>$ (consulté le 14 janvier 2013). Le site rassemble une biographie, des nouvelles sur les créations et essais du compositeur (depuis 1983), ainsi qu'un catalogue et une bibliographie d'écrits lui étant dédiés. Un examen de certaines œuvres peut être trouvé dans la publication de Lidia Bramani (mai 1996): "Marco Stroppa ", Résonance, n 10 , Ircam - Centre Georges-Pompidou. On citera également le travail de Giacomo Albert (2006): "La musica di Marco Stroppa: percorsi paralleli tra tecnologia e pensiero compositivo", mémoire de maîtrise, Université de Pavie. Enfin, le texte de Florian Hoelscher (2009) présente quelques réflexions plus récentes: "Anmerkungen zu Marco Stroppas Zyklus miniature estrose für pianoforte d'amore", Max-Planck-Institut für Wissenschaftsgeschichte, Preprint 366, p. 19-24; disponible en ligne: $<$ www.mpiwg-berlin.mpg.de/Preprints/P366.pdf > consulté le 14 janvier 2013). Marco Stroppa est publié par Ricordi (Milan, Italie).

6. Voir notamment le dossier pédagogique réalisé par Nathalie OttoWitwicky pour l'Opéra Comique de Paris (2012): "Re Orso: dossier pédagogique", <www.opera-comique.com/wp-content/uploads/2012/03/ Dossier-pédagogique-re-orso_OK.pdf> (consulté le 14 janvier 2013).

7. Stroppa est le premier compositeur à utiliser le mot Leitgestalten, tiré de la psychologie de la Gestalt, dans le domaine compositionnel.

8. Marco Stroppa (1989), "Musical Information Organisms: An Approach to Composition", Contemporary Music Review, vol. 4, nº 1 , p. 131-163. Traduction française: "Les organismes d'information musicale: une approche de la composition", in Stephen McAdams et Irène Déliège (dir.), La musique et les sciences cognitives, Bruxelles, Pierre Mardaga, p. 203-234. 